\title{
Mild Traumatic Brain Injury in Children: Strategies to Reduce the in Hospital Observation Period
}

\author{
Markus Lehner ${ }^{1 *}$, A Koenig ${ }^{1}$, B Schuster ${ }^{1}$, HG Dietz ${ }^{1}$ and D Wendling-Keim ${ }^{1}$ \\ ${ }^{1}$ Department of Pediatric Surgery, Dr. von Hauner Children's Hospital, Ludwig Maximillian's University, Germany
}

Submission: April 24, 2017; Published: May 08, 2017

*Corresponding author: Markus Lehner, Department of Pediatric Surgery, Children`s Hospital, Kantonsspital Luzern, 6004 Luzern, Switzerland, Email: markus.lehner@luks.ch

Abbreviations: mTBI: Mild Traumatic Brain Injury; ED: Emergency Department; MRI: Magnet Resonance Imaging;cCT: cerebral Computed Tomography; ciTBI: clinically important TBI; pGCS: pediatric Glasgow Coma Score

\section{Introduction}

Mild Traumatic Brain Injury (mTBI) in children is one of the most frequent causes for admission at our level one pediatric surgery Emergency Department (ED) unit at the Dr. von Hauner Children's Hospital, University of Munich, Germany. For evaluation of mTBI patients different strategies are common in the ED setting [1]. The observation period is still controversial [2]. Because of the higher radio-sensitivity of children X-ray exposition should be as low as reasonable achievable because of the risk of cancer [3].
Besides different imaging modalities to detect intracranial lesions - such as cranial ultrasound or magnet resonance imaging (MRI) of the head computed tomography (CT) is still the Gold standard [4,5]. To avoid radiation exposure, in our algorithm (Figure 1) we focus on the clinical neurologic status [6]. In pediatric patients undergoing radiological examination after mTBI (using MRI or CT) intracranial injuries were detected in $4-8 \%[7,8]$. Overall, less than $0.5 \%$ of these patients need a neurosurgical intervention [9-11]. The aim of the study was to evaluate our modified algorithm in treating children suffering from mild TBI.

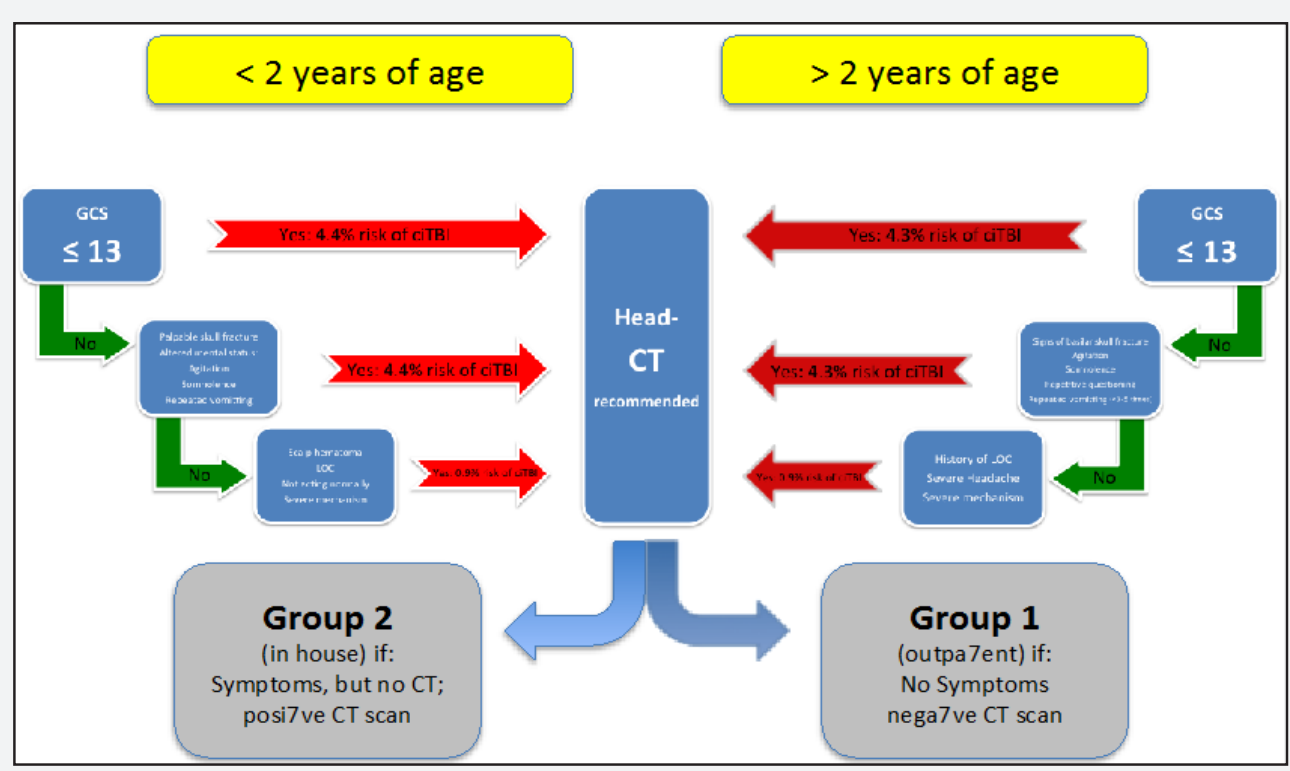

Figure 1: Algorithm. 


\section{Methods}

267 consecutive cases of children presenting to our pediatric surgery ED during July 2010 and December 2010 with clinical symptoms of mTBI were analyzed. Clinically important TBI (ciTBI) was defined as head injury resulting in intubation for $>24$ h, neurosurgery, intracranial hemorrhage without neurosurgery or more than two nights of hospitalization for the management

of mTBI in these patients, modified to Schoenfeld et al. 2015 [12].

Type of Intervention: Based on risk stratification of the clinical signs and symptoms (Figure 1, adapted PECARN recommendations; [9,13]), we analyzed retrospectively the feasibility of our algorithm. All children presenting to our ED in-between July and December 2012 were evaluated for clinical signs of mild TBI, such as impaired cognition, signs of a skull fracture, vomiting and hematoma of the scalp. Study setting: In the pediatric surgery emergency department as a level one trauma center for children almost 15.000 children $<18$ years were treated per year. No ED observation unit is available. Typically, residents in the fourth to sixth year of training in pediatric surgery are responsible for the ED. They evaluate all of the patients and are responsible for their diagnostic and therapeutic strategies. The adapted PECARN guideline of our institution is shown in Figure 1 [12,13]. Inclusion criteria were an accident and consecutive clinical signs of mTBI under the age of 18 years presenting 13-18 points in the pGCS in the ED. Pediatric Glasgow Coma Score (pGCS) in the ED between 13 - 15.

Exclusion criterion was a GCS below 13 when presenting first to our ED. Participants: all patients presenting to our pediatric surgery ED, Dr. von Hauner Children's Hospital University of Munich during July and December 2010. Statistical analyses were conducted using IBM SPSS Statistics 20.0. All analyses were realized in a PASW-Matrix (Predictive Analysis Soft Ware, SPSS Inc). Significance was analyzed with the Pearson's Chi-square test (20.0021); statistical significance was set at an alpha level of $\mathrm{p}=0.05$. All data were irreversibly made anonymous. The study protocol recently was published Wendling et al. [14].

\section{Results}

267 patients with a mean age of 49.3 months (median 33 months) were analyzed. 140 patients were out patients (Group 1) and 127 were in house patients (Group 2). 78\% of mTBI patients in our study did not receive a diagnostic imaging procedure at all. These patients did not present with pathological findings in the clinical or neurological examination in the ED and consequently their risk for a skull fracture with or without intracranial bleeding was categorized as negligibly low. None of the 267 cases detoriated into a higher graded TBI during a follow up period of 6 months. In house patients were more likely to receive diagnostic imaging procedures (Figure $2 ; \mathrm{p}=<0.05$ ).
There was no pathological finding in the 13 diagnostic studies performed in the outpatient group.

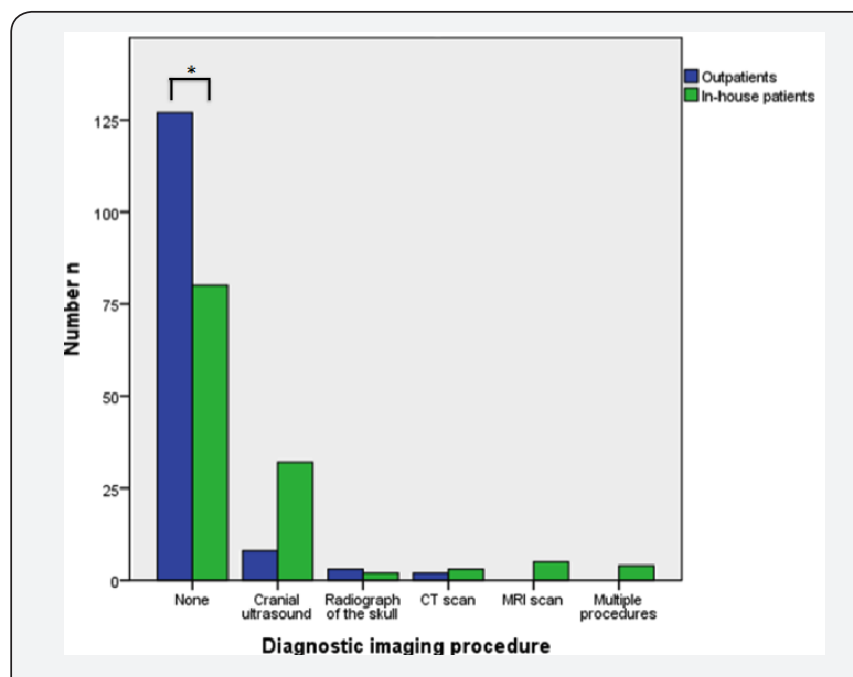

Figure 2: Diagnostic procedures $\left({ }^{*} \mathrm{p}=<0.05\right)$.

Out of 54 in house patients, 34 got a diagnostic procedure (62\%), but only $3 / 54(5.55 \%)$ were evaluated with a CT scan in this group. Out of all analyzed patients, only $5 / 267$ was analyzed with a CT scan of the head. This is a CT rate of $1.87 \%$ out of 267 patients diagnosed with mTBI, which is extremely low. None of the in house and outpatients returned after discharge to our or any other ED, based on the questionnaire [14]. Duration of the inpatient treatment was analyzed and demonstrated that patients in our study were hospitalized in $94.3 \%$ for one or two nights. The three hospitalized patients classified as ciTBI in Group 2 (in patients) were hospitalized for 5-8 days because of a skull fracture in one case and a skull fracture and intracranial hemorrhage in two cases. In one of the latter an epidural hematoma was evacuated. The $\mathrm{CCT}$ in all three cases was performed within an 8-hour period post admission to our ward.

\section{Discussion}

Palchak et al. [9] reported that $99 \%$ of the children under the age of 2 years with CT-confirmed intracranial injury could already be diagnosed by clinical signs and symptoms. The risk to develop leukemia in childhood is significantly increased by radiological studies the younger the children are [15-17], especially in the use of cCT [3]. An investigation by Thomas et al. [6] showed that the effect of ionizing radiation is underestimated by many physicians. Otherwise, in children under the age of one year occurs the highest incidence of skull fractures and intracranial injuries $[18,19]$.

Based on these findings we adapted our protocol towards a risk stratification first on the clinical signs and symptoms (Figure 1). If there were no signs of impaired cognition, signs of a skull fracture, vomiting and hematoma of the scalp the patients a CT scan of the head was not considered. For children younger 
than 2 years of age, the risk for an intracranial injury is below $0,02 \%$ [10], when there is no loss of consciousness, behavior is unchanged in the parents eyes, there has been no dangerous mechanism of injury, no hematoma of the skull and no clinical sign of a skull fracture [20]. Children older than 2 years have a risk of $0.05 \%$ for an intracranial injury if there is no loss of consciousness, no vomiting, no headache no clinical sign of a basal skull fracture and no dangerous mechanism of injury have been involved [10].

$78 \%$ of mTBI did not present with pathological findings in the clinical or neurological examination in the ED and consequently their risk for a skull fracture with or without intracranial bleeding was categorized as negligibly low. None of the 267 cases detoriated into a higher graded TBI during a follow up period of 6 months. In our algorithm clinical observation comes before radiologic examination in cases of mTBI. This is in accordance with the study published by Nigrovic et al. in 2015 [11]. Every hour of clinical evaluation increases the findings on a CT scan if the children are going to be re-evaluated. Respectively false negative scans could be diminished. In our study population we could reduce the frequency of the CT scan in infants and children suffering from mTBI down to a rate less than $2 \%$. Future speaking these patients will be clinical evaluated within the ED. This data lead to a feasible algorithm (Figure 1) and gives the opportunity to a change in the observation strategies in children suffering from mild TBI. In a lot of hospitals especially in Europe the observation period is still at least 48 hours routinely.

\section{Conclusion}

We demonstrate that our algorithm is feasible for children suffering from mTBI. Out of 267patients, none of them showed deterioration and turned back to our or any other ED because of neurologic signs of a mTBI (vomiting, altered mental status, severe headache). Children suffering from mTBI and a GCS 1315 benefit from a structured neurological observation. The main issue in mTBI should be clinical observation. Based on our data the observation period of 48 hours as performed in many institutions in Europe is controversial. In Our data indicate for an observation period less than 48 hours. 12-24 hours could be enough. Further studies in a multi-center setting should be performed. Based on these considerations the hospital invoicing system has to be adopted.

\section{References}

1. (1999) The management of minor closed head injury in children. Committee on Quality Improvement, American Academy of Pediatrics. Commission on Clinical Policies and Research, American Academy of Family Physicians. Pediatrics 104(6): 1407-1415.

2. Schnadower $D(2007)$ Controversies in the evaluation and management of minor blunt head trauma in children. Curr Opin Pediatr 19(3): 258264.
3. Pearce MS (2012) Radiation exposure from CT scans in childhood and subsequent risk of leukaemia and brain tumours: a retrospective cohort study. Lancet 380(9840): 499-505.

4. Ashwal S, Holshouser BA, Tong KA (2006) Use of advanced neuroimaging techniques in the evaluation of pediatric traumatic brain injury. Dev Neurosci 28(4-5): 309-326.

5. Osmond MH, Klassen TP, Wells GA, Correll R, Jarvis A (2010) CATCH: a clinical decision rule for the use of computed tomography in children with minor head injury. CMAJ 182(4): 341-348.

6. Thomas KE (2006) Assessment of radiation dose awareness among pediatricians. Pediatr Radiol 36(8): 823-832.

7. Nee PA (1999) Significance of vomiting after head injury. J Neurol Neurosurg Psychiatry 66(4): 470-473.

8. Glass T (2015) Traumatic brain injuries and computed tomography use in pediatric sports participants. Am J Emerg Med 33(10): 14581464.

9. Palchak MJ (2003) A decision rule for identifying children at low risk for brain injuries after blunt head trauma. Ann Emerg Med 42(4): 492506.

10. Dayan PS (2014) Risk of traumatic brain injuries in children younger than 24 months with isolated scalp hematomas. Ann Emerg Med 64(2): 153-162.

11. Nigrovic LE (2015) Quality Improvement Effort to Reduce Cranial CTs for Children With Minor Blunt Head Trauma. Pediatrics 136(1): e227233.

12. Schonfeld D (2015) Pediatric Emergency Care Applied Research Network head injury clinical prediction rules are reliable in practice. Postgrad Med J 91(1081): 634-638.

13. Bressan S (2012) Implementation of adapted PECARN decision rule for children with minor head injury in the pediatric emergency department. Acad Emerg Med 19(7): 801-807.

14. Wendling-Keim (2017) Ambulatory or inpatient management of mild TBI in children: a post-concussion analysis. Pediatr Surg Int 33(2): 249-261.

15. Brenner DJ (2002) Estimating cancer risks from pediatric CT: going from the qualitative to the quantitative. Pediatr Radiol 32(4): 228-281.

16. Brenner D (2001) Estimated risks of radiation-induced fatal cancer from pediatric CT. AJR Am J Roentgenol 176(2): 289-296.

17. Teasdale GM (1990) Risks of acute traumatic intracranial haematoma in children and adults: implications for managing head injuries. BMJ 300(6721): 363-367.

18. Kleinman PK, Spevak MR (1992) Soft tissue swelling and acute skull fractures. J Pediatr 121(5 Pt 1): 737-739.

19. Hebb MO, DB Clarke, JM Tallon (2007) Development of a provincial guideline for the acute assessment and management of adult and pediatric patients with head injuries. Can J Surg 50(3): 187-194.

20. Kuppermann (2009) Identification of children at very low risk of clinically-important brain injuries after head trauma: a prospective cohort study. Lancet 374(9696): 1160-1170. 
This work is licensed under Creative Commons Attribution 4.0 Licens DOI: 10.19080/OAJS.2017.04.555635
Your next submission with Juniper Publishers will reach you the below assets

- Quality Editorial service

- Swift Peer Review

- Reprints availability

- E-prints Service

- Manuscript Podcast for convenient understanding

- Global attainment for your research

- Manuscript accessibility in different formats

( Pdf, E-pub, Full Text, Audio)

- Unceasing customer service

Track the below URL for one-step submission https://juniperpublishers.com/online-submission.php 